\title{
Validity of a short questionnaire to assess physical activity in 10 European countries
}

\author{
The InterAct Consortium
}

Received: 16 August 2011/Accepted: 7 October 2011/Published online: 17 November 2011

(C) The Author(s) 2011. This article is published with open access at Springerlink.com

\begin{abstract}
To accurately examine associations of physical activity (PA) with disease outcomes, a valid method of assessing free-living activity is required. We examined the validity of a brief PA questionnaire (PAQ) used in the European Prospective Investigation into Cancer and Nutrition (EPIC). PA energy expenditure (PAEE) and time spent in moderate and vigorous physical activity (MVPA) was measured in 1,941 healthy individuals from 10 European countries using individually-calibrated combined heart-rate and movement sensing. Participants also completed the short EPIC-PAQ, which refers to past year's activity. Pearson (r) and Spearman $(\sigma)$ correlation coefficients were calculated for each country, and random effects meta-analysis was used to calculate the combined correlation across countries to estimate the validity of two previously- and one newly-derived ordered, categorical PA indices ("Cambridge index", "total PA index", and "recreational index") that categorized individuals as inactive, moderately inactive, moderately active, or active. The strongest associations with PAEE and MVPA were observed for the Cambridge index $(\mathrm{r}=0.33$ and $\mathrm{r}=0.25$, respectively). No significant heterogeneity by country was observed for this index $\left(\mathrm{I}^{2}=36.3 \%, P=0.12 ; \mathrm{I}^{2}=0.0 \%\right.$, $P=0.85$ ), whereas heterogeneity was suggested for other
\end{abstract}

The members of the InterAct consortium is given in Appendix.

InterAct project was coordinated by the Medical Research Council Epidemiology Unit, Cambridge, UK.

Electronic supplementary material The online version of this article (doi:10.1007/s10654-011-9625-y) contains supplementary material, which is available to authorized users.

The InterAct Consortium ( $\square)$

Cambridge, UK indices $\left(\mathrm{I}^{2}>48 \%, P<0.05, \mathrm{I}^{2}>47 \%, P<0.05\right)$. PAEE increased linearly across self-reported PA categories ( $P$ for trend $<0.001$ ), with an average difference of approximately $460 \mathrm{~kJ} / \mathrm{d}$ for men and $365 \mathrm{~kJ} / \mathrm{d}$ for women, between categories of the Cambridge index. The EPIC-PAQ is suitable for categorizing European men and women into four distinct categories of overall physical activity. The difference in PAEE between categories may be useful when estimating effect sizes from observational research.

Keywords Physical activity · Validity · Self-report . Questionnaire · Accelerometry

\section{Introduction}

Physical inactivity is a major risk factor for mortality and for several chronic diseases, including type 2 diabetes [1]. Large cohort studies are required to examine the etiology of chronic disease outcomes among healthy individuals, and in large, multi-site prospective studies of physical activity in relation to chronic disease, self-report methods such as physical activity questionnaires (PAQs) are currently the most feasible method for assessing physical activity. PAQs are commonly used for practical reasons such as limiting cost and reducing participant and researcher burden, and PAQs have been used for several purposes, including international surveillance (e.g., the International Physical Activity Questionnaire [IPAQ] [2]), risk stratification (e.g., the EPIC Physical Activity Questionnaire [EPIC-PAQ] [3]), and etiologic investigation (e.g. the short European Prospective Investigation into Cancer and Nutrition [EPIC]-Norfolk Physical Activity Questionnaire [EPAQ2] [4]). However, PAQs may misclassify an individual's physical activity level due to deliberate 
misreporting or cognitive limitations related to recall or comprehension $[5,6]$. Therefore, it is necessary to examine the validity of any self-report instrument using independent criterion methods in a population representative of that in which it is used for answering epidemiological questions.

The EPIC study was designed to investigate the relationship of nutrition and cancer in over 500,000 individuals from 10 European countries [7]. At enrolment (1992-2000), study participants completed questionnaires assessing diet and lifestyle factors, including physical activity. Specifically, physical activity was assessed by a brief questionnaire (the short EPIC-PAQ) interrogating occupational, household, and recreational activities during the past year [8].

An initial evaluation of the validity of questions selected from a more extensive questionnaire used in a pilot study of the EPIC protocol that resembled questions interrogated by the short EPIC-PAQ was performed in the Netherlands [9]. The authors assessed the validity and reliability of these representative questions against physical activity reported on 3-day diaries and determined that although the absolute validity and reliability of the questions for estimating physical activity energy expenditure (PAEE) was poor, the questionnaire would be suitable for ranking physical activity levels.

Accordingly, two indices for categorizing physical activity levels have been derived from the short EPICPAQ, the "Cambridge index" [3] and the "total physical activity index" [10]. The validity of these indices for categorizing individuals' physical activity levels has not yet been assessed in the EPIC cohort. Therefore, the aim of this study was to evaluate the validity of these indices for ranking physical activity among adults from the 10 countries participating in the EPIC-Europe study using combined heart rate (HR) and movement sensing as the criterion. We also examined the validity of a newly-derived index considering only recreational physical activity.

\section{Methods}

\section{Study population}

In each of twelve centres across ten countries (Aalborg, Denmark; Paris, France; Potsdam, Germany; Athens, Greece; Florence, Italy; Bilthoven and Utrecht, Netherlands; Troms $\varnothing$, Norway; Murcia and San Sebastian, Spain; Umeå, Sweden; Cambridge, UK), a sample of approximately 200 healthy individuals of a centre-specific age and gender distribution similar to that of the original EPIC-Europe cohort [7] was recruited. In accordance with the original EPIC-Europe design, healthy, middle-aged men and women were recruited in all centres, with the exception of France and Norway, where only women were included.
The response rate varied across study centres from $37 \%$ in Norway to $97 \%$ in Italy, and retention of enrolled participants for the duration of the study was $\geq 87 \%$ in all study centres.

\section{Study design}

The study consisted of two visits held four to 5 months apart (mean time between visits $=4.53$ months; $\mathrm{SD}=1.02$ ) (Fig. 1). At each visit, height was measured with a rigid, portable stadiometer, and weight was measured using a standard scale or a bio-impedance scale $\left(\right.$ Tanita $\left.^{\circledR}\right)$. Participants completed a general questionnaire that merged the Rose Angina Questionnaire [11] and the Physical Activity Readiness Questionnaire [12] to determine eligibility to engage in a sub-maximal physical activity calibration test (step test). In some study centres, a resting electro-cardiogram (ECG) was administered as an additional safety measure.

To standardize the study protocol across centres, the MRC Epidemiology Unit held a 2-day training workshop in Cambridge, UK, for the field workers from each study location (March 2007). For quality control, MRC Epidemiology Unit staff also visited study centres during the testing phase (March 2007-December 2008). Each centre obtained ethical approval from a local ethics board prior to participant recruitment, and informed consent was obtained from all participants.

\section{Objective physical activity measurement methods}

Eligible participants were fitted with a combined HR and movement sensor (Actiheart, CamNtech, Cambridge, UK), which was attached to the chest via two standard ECG electrodes [13]. An 8-min, ramped step test using a

RECRUITMENT

- 200 participants per country

- Similar in age and gender distribution as original EPIC cohort

FRIST VISIT

- Measurements of height, weight, waist and hip circumference

- Step test for individual calibration of heart rate vs. work load relationship

- Free-living assessment of physical activity energy expenditure by

combined movement and heart rate sensing

SECOND VISIT ( 4 months after first visit)

- Measurements of height, weight, waist and hip circumference

- Step test for individual calibration of heart rate vs. work load relationship

- Administration of EPIC-PAQ

- Free-living assessment of physical activity energy expenditure by

combined movement and heart rate sensing

Fig. 1 Study design: the EPIC-PAQ validation study 
200-mm step (Reebok, Lancaster, UK) was then performed to determine the individual relationship between HR and workload [14]. The equation for the relationship between PAEE and heart rate from the step test (3865 tests) was as follows;

$\mathrm{PAEE}[\mathrm{J} / \mathrm{min} / \mathrm{kg}]=(6.22-0.003 *$ age $+0.28 * \mathrm{sex}-$ $0.0062 * \mathrm{SHR}) * \mathrm{HRaS}+0.21 *$ age $+3.9 *$ sex $-0.97 *$ SHR-31.8 (age in yrs, sex coded as 1 for men and 0 for women, SHR is sleeping HR in bpm, HRaS is HR above SHR in bpm).

Specifically, participants were asked to step up and down following a timed voice prompt at a step frequency that began at 15 body lifts per minute (60 steps/min) and increased linearly to a maximum of 33 lifts per minute, immediately followed by a 2-min seated recovery phase. Following the step test, the combined HR and movement sensor was initialized for long-term recording summarized into 1-min epochs, and participants were instructed to wear the monitor continuously for a minimum of 4 days of freeliving data collection.

Data collected during free-living was downloaded to a $\mathrm{PC}$ and the HR trace was processed using a robust Gaussian Process regression method to handle potential measurement noise [15]. Activity intensity $(\mathrm{J} / \mathrm{min} / \mathrm{kg})$ for each time point was estimated from the combination of movement registration and individually calibrated HR [14] using a branched equation framework [16]. Periods of non-wear were inferred from the combination of non-physiological HR and prolonged periods of inactivity, which were taken into account to minimize diurnal information bias when summarizing the intensity time-series into PAEE $(\mathrm{kJ} / \mathrm{kg} /$ day) and time spent in moderate-to-vigorous intensity physical activity (minutes of MVPA/day). The intensity threshold for MVPA was set at 3 metabolic equivalent task units (METs), with 1 MET defined according to the Oxford resting metabolic rate equations [17].

Finally, we excluded measurement periods with less than $24 \mathrm{~h}$ of data and averaged daily estimates of PAEE and of MVPA from the two 4-day measurements. 11 individuals ( 9 women and 2 men) were excluded as they did not provide valid data. PA outcomes were weighted to account for divergence from the optimum monitoring duration of two 4-day measurement periods (i.e., individuals with at least $4+4$ days were weighted 1.0, whereas those with fewer days were weighted less, e.g. an individual with $4+3$ days was weighted 7/8).

\section{Self-report physical activity methods (EPIC-PAQ)}

The second visit followed the same protocol as the first visit, with the additional administration of the short EPICPAQ [3]. The English version of this PAQ has been published previously $[3,10]$. Prior to objective monitoring of free-living physical activity, participants completed the EPIC-PAQ, which was administered by each centre in a format similar to the original EPIC administration, i.e., self-report (Denmark, France, Italy, the Netherlands, Norway, and the UK) or interview (Germany, Greece, San Sebastian and Murcia [Spain]). The PAQ administered in Sweden differed slightly from the EPIC-PAQ completed at other sites and is described separately (see below).

The EPIC-PAQ is comprised of four questions interrogating physical activity during the last year. The first question asks participants to indicate which category of occupational activity (sedentary, standing, manual work, heavy manual work) best defines their current job. As the EPIC-PAQs in Denmark and Sweden allowed for multiple responses, responses were collapsed into the four occupational activity categories above by taking the roundeddown average of all selected occupations, if any. Question two interrogates participation in several activities (walking, cycling, do-it-yourself [DIY], gardening, sports, and household chores) during both summer and winter, the third question asks about participation in vigorous non-occupational activities, and the fourth question assesses the number of floors of stairs climbed up per day, all referencing the past year.

From responses to these questions, participants were categorized into four physical activity levels (inactive, moderately inactive, moderately active, active) using the previously developed "total physical activity index" based on occupational activity and the duration of time spent in household chores and recreational activity [10] and the "Cambridge index" based on occupational activity and the duration of time spent in sports and cycling [3], as well as a newly developed "recreational index" (Table 1), as follows.

We computed the total physical activity index according to previous recommendations [3, 10]. Briefly, to denote activity intensity, we assigned MET-values using standard methods [18]: 3.0 for walking and household activities; 4.0 for gardening; 4.5 for DIY work; 6.0 for cycling and sports; 8.0 for stair climbing; 9.0 for vigorous activity. To assign participants into one of the four physical activity levels of the total physical activity index, we cross-tabulated the four occupational categories with quartiles of sex-specific MET-h/week of total household and recreational activity, calculated as the sum duration of each activity from question two, averaged for summer and winter and multiplied by the corresponding MET-value.

The Cambridge index includes four categories of the sum duration of cycling and sports (h/week), cross-tabulated with occupational physical activity categories to assign participants into one of the four physical activity levels [3]. In addition, we calculated a recreational index based on quartiles of total MET-h/week from walking, 
Table 1 Definitions of physical activity indices from the European Investigation into Cancer and Nutrition-Physical Activity Questionnaire (EPIC-PAQ)

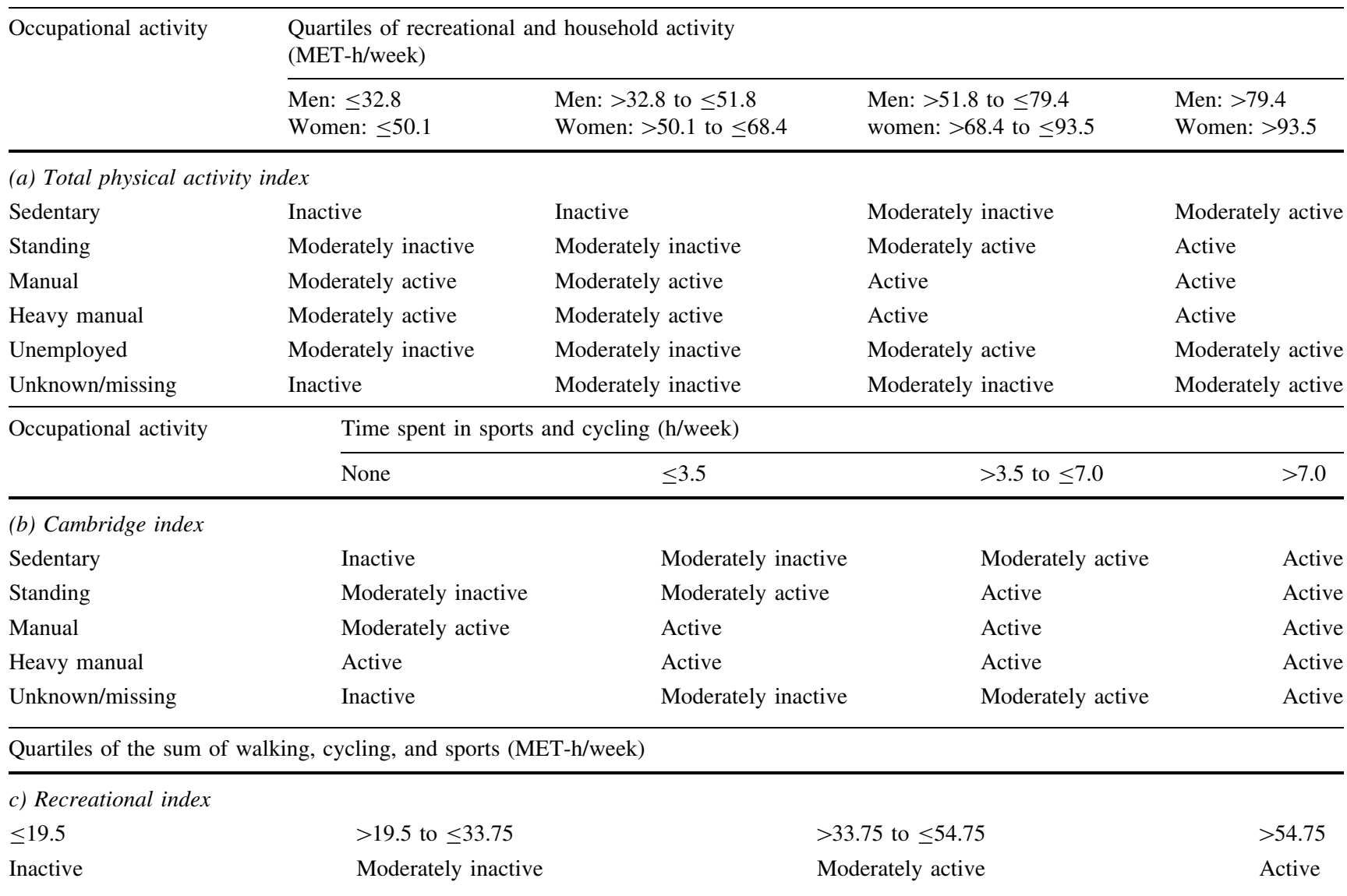

cycling, and sports activities, to reflect modifiable physical activities.

In Umeå (Sweden), the PAQ inquires about occupational and leisure time activity during the past 3 months. Due to differences in this PAQ relative to the other EPIC centres, we did not compute a total physical activity index for this centre. The Umeå index was therefore based on the cross-tabulation of occupation (4 categories) and exercise (collapsed from 5 into 4 categories: never/non-regular; 1-2 times/week; 2-3 times/week; >2-3 times/week), which categorized individuals into one of four physical activity categories similar to the Cambridge index (inactive, moderately inactive, moderately active, active). Finally, the "recreational index" in Umeå was based on responses to the question on exercise participation.

\section{Statistical methods}

We present the characteristics of participants in each country using means and standard deviations for continuous variables and frequencies and percentages for categorical variables. Physical activity from the combined HR and movement sensor is described as medians and inter- quartile ranges (IQR), and the Kruskal-Wallis test was used to determine differences in physical activity by gender and by country.

For each country, the Pearson (r) and Spearman $(\sigma)$ correlation coefficients were used to examine correlations of the total physical activity index, the Cambridge index, and the recreational index with PAEE and MVPA measured by the combined HR and movement sensor. Fishertransformed correlations were estimated for each country, and random effects meta-analysis methods were used to calculate the combined correlation across countries. Heterogeneity across countries in the association of each physical activity index with PAEE and MVPA was evaluated by Forest plots and was assessed using the I-squared $\left(\mathrm{I}^{2}\right)$ statistic. To examine heterogeneity in the association of each physical activity index with log-transformed PAEE and MVPA by age, gender, body mass index (BMI), and occupational status, we added the corresponding interaction term in multiple linear regression models and tested the significance of the interaction term.

We assessed the ability of the three indices to rank physical activity by calculating the mean PAEE and mean MVPA from the HR and movement sensor per activity 
category and then evaluating the $P$ value for linear trend by general linear modelling across the four categories of the index. For each index, we also determined the mean energy difference $(\mathrm{kJ} / \mathrm{day})$ between each category of the index. All statistical tests were two-sided with significance defined as a $P$ value $<0.05$, and all analyses were performed using SAS version 9.1 (Cary, North Carolina) except for the random effects meta-analysis, which was performed using STATA version 10.0 (StataCorp, College Station, TX).

\section{Results}

The study population included 1,941 participants with an average age of 53.8 years $(\mathrm{SD}=9.4)$ and an average BMI of $25.8 \mathrm{~kg} / \mathrm{m}^{2}(\mathrm{SD}=4.1)$. Women comprised the majority of the study population $(70 \%)$, and they were younger $(P=0.018)$ and leaner $(P<0.001)$ than the men (Table 2). The mean age of participants at baseline varied across countries, ranging from 49.8 to 61.1 years for men and from 47.6 to 59.4 years for women. Compared with other countries, mean BMI was lowest among men and women from the Netherlands $\left(23.5\right.$ and $22.6 \mathrm{~kg} / \mathrm{m}^{2}$, respectively), while men and women from Greece had the highest mean BMI $\left(27.8\right.$ and $27.0 \mathrm{~kg} / \mathrm{m}^{2}$, respectively).

On average, participants wore the combined HR and movement sensor for 4.9 days $(\mathrm{SD}=1.11)$ and 4.8 days $(\mathrm{SD}=1.10)$ during the two respective measurement periods. Table 3 displays the median PAEE and time spent in MVPA. We observed heterogeneity in PAEE and MVPA across countries $(P<0.001)$, with the greatest PAEE among men in the Netherlands (median PAEE $=54 \mathrm{~kJ} / \mathrm{kg}$ / day) and women in Spain (median PAEE $=46 \mathrm{~kJ} / \mathrm{kg} / \mathrm{day}$ ) and the most time spent in MVPA among men in Sweden (median MVPA $=112 \mathrm{~min} /$ day) and women in the Netherlands (median MVPA $=88 \mathrm{~min} /$ day). Overall, PAEE $(P$ value $<0.001)$ and time spent in MVPA $(P$ value $<0.001$ ) was greater for men than women.

Over $40 \%$ of participants reported working in a sedentary occupation, and a substantial proportion of participants in Greece $(45 \%)$ and the Netherlands (48\%) were categorized as unemployed (retired or not currently working) (data not shown). Overall, women reported spending more time walking, in sports, and doing housework $(P<0.05)$ compared with men, while men spent more time than women in home improvement activities $(P<0.001)$ (data not shown).

Figure 2 shows the correlations between PAEE, MVPA, and the three self-reported indices of physical activity. Overall, we observed stronger correlations between PAEE and the Cambridge index $(\mathrm{r}=0.33,95 \%$ CI $0.28,0.38)$ compared with the total physical activity index $(r=0.14$, $95 \%$ CI $0.04,0.24)$ and the recreational index $(r=0.22$,
95\% CI 0.16,0.28). We observed significant heterogeneity across countries in the correlations of the total physical activity index $\left(\mathrm{I}^{2}=80.5 \%, P<0.001\right)$ and the recreational index $\left(\mathrm{I}^{2}=48.5 \%, P=0.04\right)$, but not the Cambridge index $\left(\mathrm{I}^{2}=36.3 \%, P=0.12\right)$. The overall correlation with time spent in MVPA was slightly stronger for the Cambridge index $(\mathrm{r}=0.25$ [95\% CI $0.21,0.29$ ] vs. $\mathrm{r}=$ 0.23 [95\% CI $0.17,0.29]$ and $\mathrm{r}=0.11$ [95\% CI $0.01,0.21]$ for the recreational and total physical activity indices, respectively);only associations of MVPA with the Cambridge index did not appear heterogeneous across countries $\left(\mathrm{I}^{2}=0.0 \%, P=0.85\right)$.

In general, Pearson and Spearman correlation coefficients were similar in magnitude across study locations (Supplemental Table 1). Associations of PAEE and MVPA with the total physical activity index and the Cambridge index showed statistically significant interactions with sex ( $P$ value for interaction $<0.001$ ), whereas associations with the recreational index did not differ significantly between men and women $(P$ value for interaction $=0.38$ and 0.25 for PAEE and MVPA, respectively). We did not observe heterogeneity in the association of PAEE or MVPA with any of the indices by occupational status.

Each physical activity index was positively associated with PAEE (Fig. 3), effectively ranking participants into levels of physical activity. Results were similar for men and women, and statistically significant trends were also observed for the association of each index with MVPA (Supplemental Table 2). We observed some variability in the difference in PAEE (kJ/day, adjusted for body weight) between levels of each physical activity index. The average absolute difference between categories for men and women, respectively, was 283 and $225 \mathrm{~kJ} /$ day for the total physical activity index, 457 and $364 \mathrm{~kJ} /$ day for the Cambridge index, and 247 and $197 \mathrm{~kJ} /$ day for the recreational index.

\section{Discussion}

The EPIC-PAQ is suitable for assessing habitual physical activity levels of European populations. All three indices were all capable of ranking individuals into levels of PAEE and MVPA. Furthermore, the physical activity indices derived from the EPIC-PAQ performed similarly among adults of the age range (18-92 years) and BMI range $\left(B M I=16.2-46.5 \mathrm{~kg} / \mathrm{m}^{2}\right)$ included in our study population, and validity of the indices did not vary by employment status.

Of the three indices evaluated, the Cambridge index appeared to provide stronger associations with objectivelymeasured PAEE and did not show heterogeneity across European populations. The heterogeneity for the total PA 
Table 2 Participant characteristics at baseline (mean, SD [standard deviation]), the EPIC-PAQ Validation study cohort $(\mathrm{N}=1,941)$

\begin{tabular}{|c|c|c|c|c|c|c|c|c|c|}
\hline Country & $\mathrm{N}$ & Age (years) & SD & Height (m) & SD & Weight $(\mathrm{kg})$ & SD & BMI $\left(\mathrm{kg} / \mathrm{m}^{2}\right)$ & SD \\
\hline \multicolumn{10}{|l|}{ Men } \\
\hline Denmark & 68 & 58.0 & 3.6 & 1.8 & 0.06 & 87.3 & 10.6 & 27.7 & 3.3 \\
\hline \multicolumn{10}{|l|}{ France } \\
\hline Germany & 83 & 57.5 & 3.1 & 1.8 & 0.05 & 86.2 & 12.5 & 27.6 & 3.5 \\
\hline Greece & 67 & 50.0 & 18.7 & 1.7 & 0.08 & 84.8 & 12.5 & 27.8 & 3.7 \\
\hline Italy & 53 & 52.8 & 6.6 & 1.7 & 0.06 & 78.9 & 13.6 & 26.1 & 4.0 \\
\hline Netherlands & 30 & 49.8 & 11.2 & 1.8 & 0.06 & 77.9 & 9.9 & 23.5 & 2.2 \\
\hline \multicolumn{10}{|l|}{ Norway } \\
\hline Spain & 92 & 51.2 & 7.2 & 1.7 & 0.07 & 80.5 & 11.0 & 27.1 & 3.4 \\
\hline Sweden & 98 & 52.0 & 8.0 & 1.8 & 0.07 & 85.2 & 13.1 & 26.5 & 3.6 \\
\hline United Kingdom & 100 & 61.1 & 7.8 & 1.8 & 0.06 & 85.8 & 12.4 & 27.6 & 3.3 \\
\hline Total & 591 & 54.6 & 9.8 & 1.8 & 0.07 & 84.0 & 12.4 & 27.0 & 3.6 \\
\hline \multicolumn{10}{|l|}{ Women } \\
\hline Denmark & 115 & 57.0 & 4.2 & 1.6 & 0.06 & 69.8 & 12.8 & $26.0^{\mathrm{b}}$ & 4.4 \\
\hline France & 174 & 54.2 & 7.5 & 1.6 & 0.06 & 61.3 & 9.4 & 23.2 & 3.3 \\
\hline Germany & 125 & $54.9^{\mathrm{a}}$ & 4.6 & 1.6 & 0.06 & 69.1 & 11.1 & $25.8^{\mathrm{b}}$ & 4.1 \\
\hline Greece & 121 & 51.2 & 16.0 & 1.6 & 0.06 & 69.2 & 13.1 & 27.0 & 5.4 \\
\hline Italy & 142 & 52.5 & 6.5 & 1.6 & 0.06 & 63.6 & 10.4 & 24.9 & 3.8 \\
\hline Netherlands & 183 & $58.4^{\mathrm{a}}$ & 10.3 & 1.7 & 0.06 & 62.8 & 7.9 & 22.6 & 2.3 \\
\hline Norway & 178 & 47.6 & 4.4 & 1.6 & 0.06 & 70.8 & 10.9 & 26.1 & 3.5 \\
\hline Spain & 113 & $48.6^{\mathrm{a}}$ & 8.4 & 1.6 & 0.06 & 64.9 & 9.8 & $25.4^{\mathrm{b}}$ & 3.7 \\
\hline Sweden & 96 & 51.8 & 8.5 & 1.6 & 0.06 & 72.6 & 12.9 & 26.8 & 5.0 \\
\hline United Kingdom & 103 & 59.4 & 7.6 & 1.6 & 0.06 & 70.0 & 11.3 & 26.8 & 4.1 \\
\hline Total & 1350 & $53.5^{\mathrm{a}}$ & 9.2 & 1.6 & 0.06 & 66.9 & 11.4 & $25.2^{\mathrm{b}}$ & 4.2 \\
\hline
\end{tabular}

EPIC-PAQ European Investigation into Cancer and Nutrition-Physical Activity Questionnaire

$B M I=$ body mass index

$P$ value for difference in height and weight between men and women $<0.001$ across countries

${ }^{a} P$ value for difference in age between men and women $<0.05$

${ }^{\mathrm{b}} P$ value for difference in BMI between men and women $<0.05$

index was mainly driven by results from Netherlands and France. We can only speculate why the total PA index performed less well in these countries. One possible explanation may include a systematic over-reporting of home activities, which is part of the total PA index, in low active individuals as measured by the criterion method. The heterogeneity observed for the recreational index was driven by the low correlation observed in the UK sample. This was partly explained by the low prevalence of participation in cycling and sport in the UK population.

The results for the Cambridge index correspond with a recent validation study of the short EPIC-PAQ in an Australian population, which observed slightly better correlation of the Cambridge index than the total physical activity index with accelerometer-measured physical activity [10].

A number of studies have demonstrated the utility of the Cambridge index in observational research. In a recent cross-sectional study of men and women from nine European countries [19], the Cambridge index was inversely associated with BMI and waist-to-hip ratio. In a prospective follow-up, this index predicted gain in waist circumference [20] and was also predictive of increased mortality in a prospective study of UK adults [21]. The results of our validation study confirm that the Cambridge index suitably ranks participants' physical activity levels in etiologic study settings.

Previous studies also investigated the criterion validity of the short EPIC-PAQ in selected European populations. Pols et al. [9] found that questions from the extensive EPIC-PAQ representative of the short EPIC-PAQ questions were valid when compared with physical activity diaries $(r=0.26-0.81)$ among 126 Dutch men and women aged 20-70 years. However, activity diaries rely upon selfreporting of physical activity in a manner similar to PAQs, and therefore may not represent a suitable criterion method for assessing the validity of a PAQ since the measurement 
Table 3 Physical activity energy expenditure (PAEE, $\mathrm{kJ} / \mathrm{kg} /$ day) and moderate-to-vigorous activity (min/day) from the combined heart rate monitor and movement sensing

\begin{tabular}{|c|c|c|c|c|c|c|c|c|c|c|c|c|c|c|}
\hline \multirow[t]{3}{*}{ Country } & \multicolumn{7}{|c|}{ PAEE (kJ/kg/day) } & \multicolumn{7}{|c|}{ Moderate-to-vigorous activity (min/day) } \\
\hline & \multicolumn{3}{|c|}{ Men $(\mathrm{n}=591)$} & \multicolumn{3}{|c|}{ Women $(n=1350)$} & \multirow[t]{2}{*}{$P$ value* } & \multicolumn{3}{|c|}{ Men $(\mathrm{n}=591)$} & \multicolumn{3}{|c|}{ Women $(\mathrm{n}=1350)$} & \multirow[t]{2}{*}{$P$ value* } \\
\hline & Median & IQR & & Median & $\mathrm{IQR}$ & & & Median & IQR & & Median & IQR & & \\
\hline Denmark & 42.1 & 30.5 & 52.9 & 38.0 & 30.2 & 47.3 & 0.11 & 75.5 & 43.3 & 113.5 & 63.5 & 45.0 & 96.1 & 0.28 \\
\hline France & & & & 37.5 & 30.8 & 45.4 & & & & & 65.4 & 46.3 & 88.8 & \\
\hline Germany & 41.0 & 31.3 & 49.4 & 38.5 & 30.1 & 49.5 & 0.57 & 77.5 & 50.5 & 109.8 & 73.2 & 46.6 & 101.6 & 0.42 \\
\hline Greece & 43.8 & 29.4 & 54.7 & 38.2 & 29.7 & 48.1 & 0.12 & 82.5 & 38.3 & 137.2 & 68.1 & 42.9 & 92.3 & 0.05 \\
\hline Italy & 48.7 & 41.5 & 60.1 & 44.3 & 36.8 & 55.5 & 0.04 & 81.0 & 56.4 & 113.5 & 72.5 & 46.0 & 101.0 & 0.16 \\
\hline Netherlands & 54.0 & 44.6 & 63.7 & 43.0 & 34.5 & 54.0 & 0.002 & 101.8 & 80.9 & 138.5 & 88.3 & 54.8 & 122.7 & 0.07 \\
\hline Norway & & & & 42.7 & 34.8 & 53.7 & & & & & 79.2 & 53.3 & 119.2 & \\
\hline Spain & 48.8 & 38.9 & 62.5 & 46.0 & 38.3 & 57.4 & 0.29 & 92.8 & 62.9 & 139.2 & 87.8 & 58.3 & 125.5 & 0.47 \\
\hline Sweden & 52.4 & 41.9 & 66.4 & 40.7 & 32.0 & 51.4 & $<0.001$ & 112.2 & 73.9 & 165.7 & 86.1 & 54.8 & 119.5 & $<0.001$ \\
\hline United Kingdom & 36.4 & 29.2 & 48.3 & 34.1 & 26.4 & 44.2 & 0.05 & 61.1 & 38.4 & 105.6 & 51.4 & 29.2 & 84.5 & 0.03 \\
\hline TOTAL & 44.0 & 33.4 & 55.9 & 40.5 & 32.3 & 50.6 & 0.002 & 80.8 & 49.5 & 121.0 & 72.5 & 46.9 & 105.7 & 0.013 \\
\hline$P$ value** & $<0.001$ & & & & & & & $<0.001$ & & & & & & \\
\hline
\end{tabular}

PAEE physical activity energy expenditure

$I Q R$ inter-quartile range

* $P$ value for Kruskal-Wallis test of the difference between men and women

** $P$ value for Kruskal-Wallis test of the difference across countries

error associated with diaries may be correlated with the error from the PAQ.

In the UK, the Cambridge index was shown to correlate with objectively measured physical activity assessed by four repeat periods of 4-day HR monitoring $(r=0.28)$ in 173 middle-aged participants [3]. Objective methods such as HR monitoring and accelerometry avoid the issue of shared sources of error and bias seen with activity diaries, but these methods also have limitations in PAQ validation studies. For example, current objective methods do not identify the type of physical activity nor the context in which it is performed, and therefore cannot investigate the validity of specific domains of physical activity, which would be possible using diaries. In addition, HR monitoring is a less valid measure of energy expenditure during sedentary and light activity because HR may be influenced by factors other than body movement, e.g. ambient temperature, emotional stress [22]. Similarly, a single accelerometer attached to the waist or trunk is less accurate for measuring energy expenditure associated with certain activities such as cycling, swimming, or upper-body movements.

Combined HR and movement sensing has emerged as a method to circumvent some of the limitations of the two respective methods and is a valid criterion measure for quantifying PAEE [23-25]. Integrating HR with accelerometry offers benefits over the methods used as criterion measures of physical activity in previous validation studies of the EPIC-PAQ [4, 9, 10]. In addition, the combined sensor is worn continuously to avoid exclusion of waterbased activities and sleeping time (as is often the case when using only an accelerometer on a waist belt). Precision of the energy expenditure estimate is further enhanced by individual calibration of the HR response to exercise $[14,26]$.

The estimated difference in measured PAEE, which effectively separated the four categories of self-reported activity from the Cambridge index, was about 460 and $365 \mathrm{~kJ} / \mathrm{D}$ in men and women, respectively. Although these results should be interpreted cautiously, the observed difference is equal to the PAEE associated with about $20 \mathrm{~min}$ of brisk walking and may be useful when estimating effect sizes in observational research from studies using the short EPIC-PAQ.

Our study was conducted in a population selected to be representative of the original EPIC-Europe cohort within which the EPIC-PAQ was administered at baseline. The large size of our study population $(\mathrm{N}=1,941)$, the inclusion of participants from ten European countries, and the standardization of study methods across countries are among the strengths of our study. Furthermore, we measured physical activity by combined HR and movement sensing for $\geq 4$ days on two occasions separated by $4-5$ months, and thus likely captured a wide range of usual physical activity patterns across European populations.

However, daily variability in physical activity, particularly differences between weekdays and weekend days, 
(a)Total Physical Activity Index

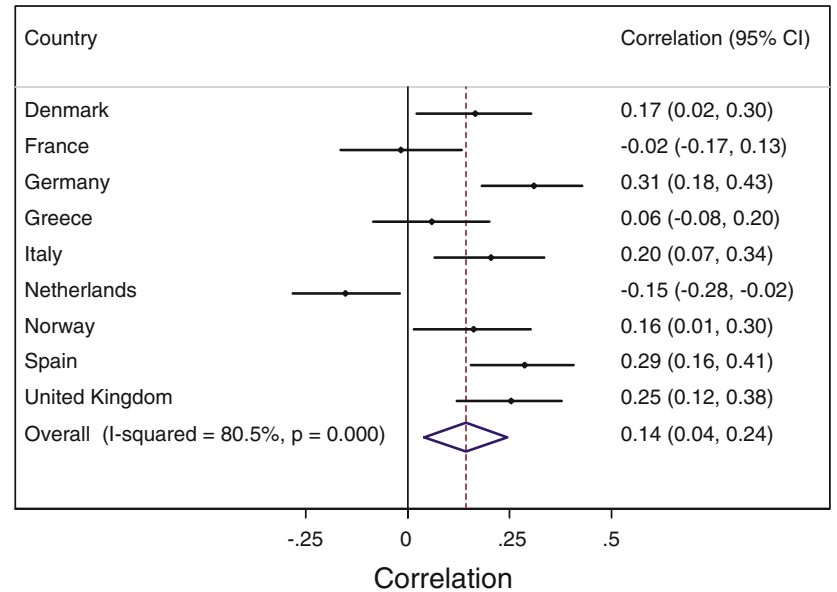

(c) Recreational Index

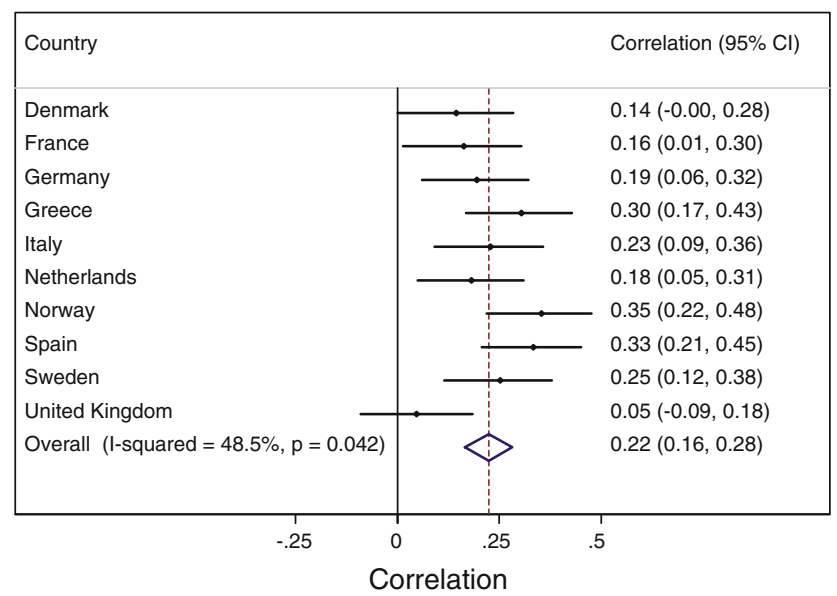

(e) Cambridge Index

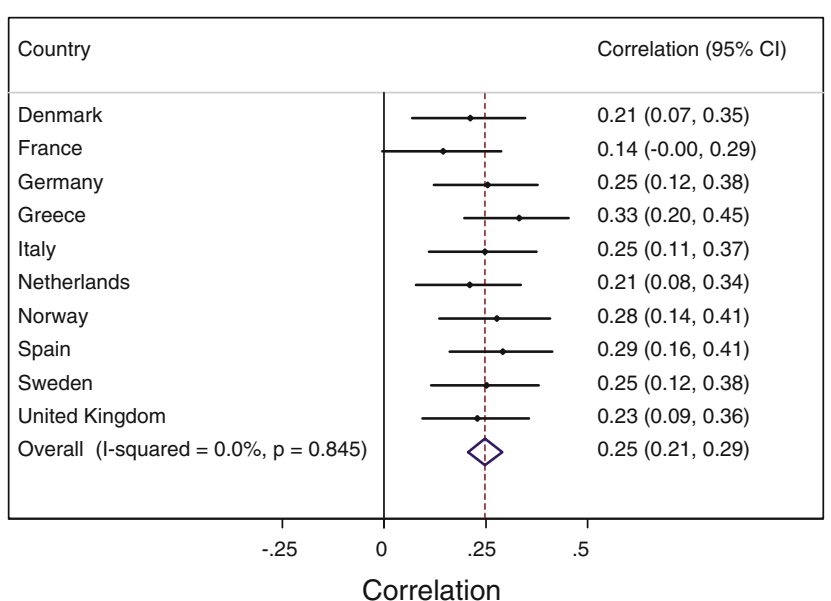

(b) Cambridge Index

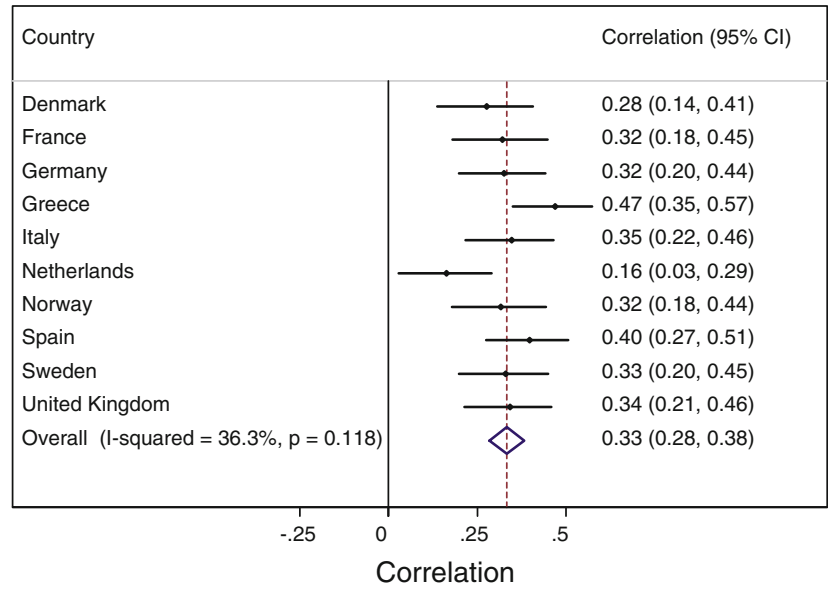

(d) Total Physical Activity Index

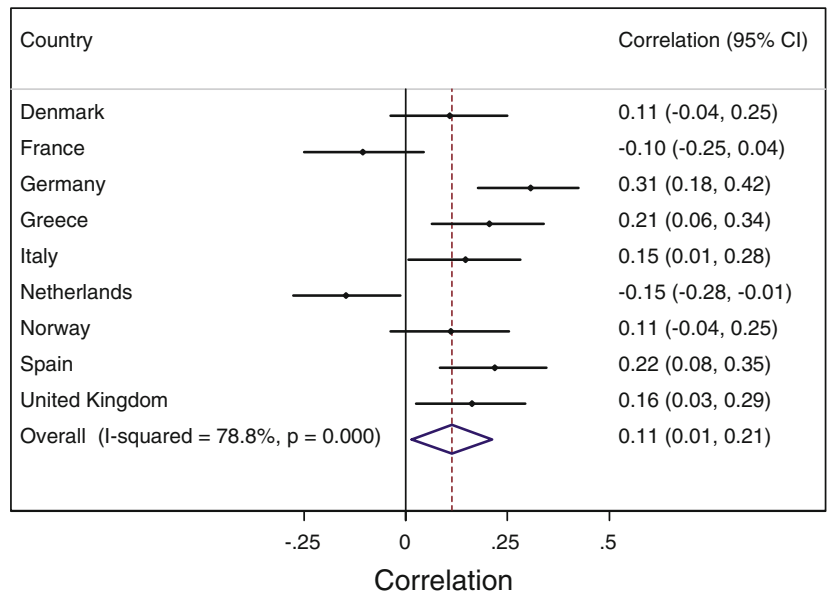

(f) Recreational Index

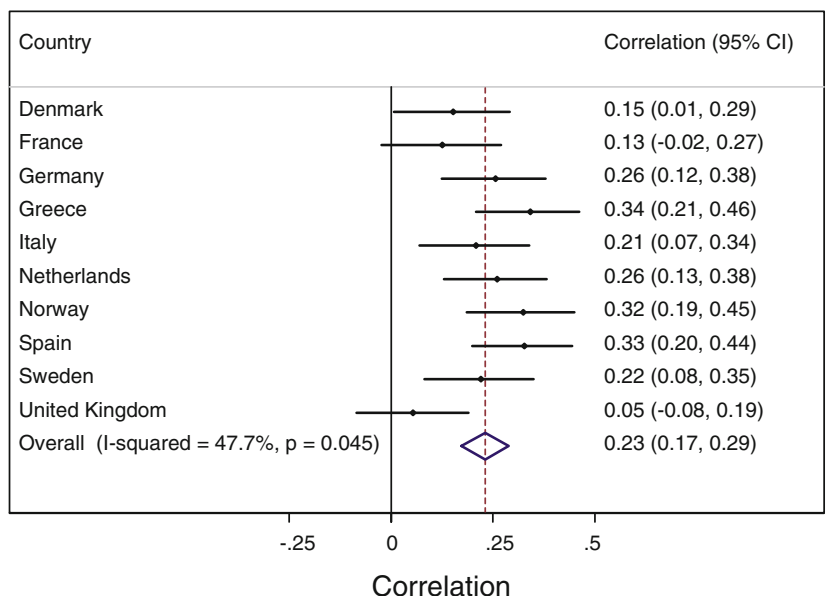

Fig. 2 Meta-analysis (Forest plots) of the association (Pearson correlation coefficients) of PAEE (a-c) and moderate-to-vigorous activity (d-f) measured by the combined HR and movement sensor with three physical activity indices from the EPIC-PAQ, by country and overall 


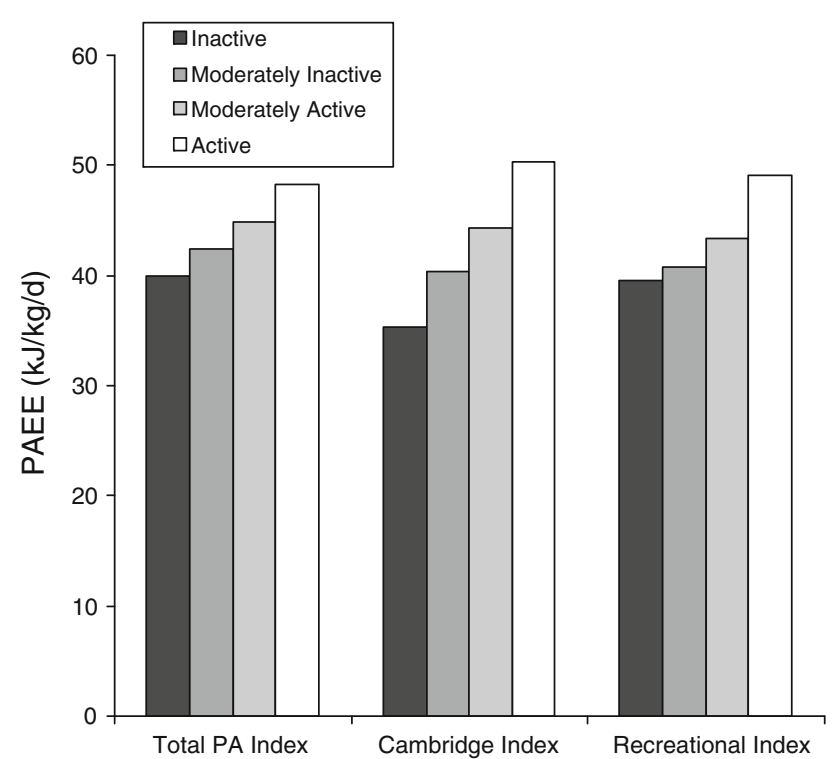

Fig. 3 Mean PAEE (kJ/kg/day) from the combined HR and movement sensor stratified by each physical activity category $(1=$ inactive, $2=$ moderately inactive, $3=$ moderately active, $4=$ active) from the short EPIC-PAQ ( $a$ "Total physical activity index"; $b$ "Cambridge index"; $c$ "Recreational index")

might have been better captured by one full week of physical activity monitoring, and within-individual seasonal differences in physical activity would be better assessed by more than two measurement periods [27]. Another limitation of our study includes the potential misclassification of participants' physical activity levels based on occupational physical activity, as individuals who did not report current occupational activity were included in the lowest occupational activity category for the Cambridge index (Table $1 b$ ). However, correlations of the Cambridge index with objectively-assessed PAEE and MVPA were similar $(r=0.32$ and $\mathrm{r}=0.26$, respectively) when we excluded participants with no reported occupational activity.

In conclusion, the EPIC-PAQ provides a brief, global assessment of habitual physical activity, and can be used to discriminate between levels of usual physical activity by means of the physical activity indices herein described. Our results suggest that the EPIC-PAQ, and the Cambridge index in particular, is suitable for ranking habitual physical activity of individuals across European populations. The difference in PAEE between categories may be useful when estimating effect sizes from observational research.

Acknowledgments We are grateful to all participants who gave their time and effort to the study. We are also extremely grateful to all persons who contributed to the data collection across the study sites. A special thank to the MRC Epidemiology Unit physical activity technical team; Mark Betts, Laura Lamming and Stefanie Mayle who assisted with data reduction, cleaning and processing. Your efforts are highly appreciated. This study was supported by funding from the
European Union (Integrated Project LSHM-CT-2006-037197 in the Framework Programme 6 of the European Community) and the Medical Research Council, UK.

\section{Conflict of interest None.}

Open Access This article is distributed under the terms of the Creative Commons Attribution Noncommercial License which permits any noncommercial use, distribution, and reproduction in any medium, provided the original author(s) and source are credited.

\section{Appendix}

\section{Author List}

Tricia Peters ${ }^{1}$, Soren Brage ${ }^{1}$, Kate Westgate ${ }^{1}$, Paul W Franks $^{2,15}$, Anna Gradmark ${ }^{2}$, Maria Jose Tormo Diaz ${ }^{3,4}$, Jose Maria Huerta ${ }^{3,4}$, Benedetta Bendinelli ${ }^{5}$, Mattheaus Vigl $^{6}$, Heiner Boeing ${ }^{6}$, Wanda Wendel-Vos ${ }^{7}$, Annemieke Spijkerman $^{7}$, Kristin Benjaminsen- Borch ${ }^{8}$, Elisavet Valanou $^{9}$, Blandine de Lauzon Guillain ${ }^{10}$, Françoise ClavelChapelon ${ }^{10}$, Stephen Sharp ${ }^{1}$, Nicola Kerrison ${ }^{1}$, Claudia Langenberg $^{1}$, Larraitz Arriola ${ }^{11}$, Aurelio Barricarte ${ }^{4,12}$, Carlos Gonzales ${ }^{13}$, Sara Grioni ${ }^{14}$, Rudolf Kaaks ${ }^{15}$, Timothy Key ${ }^{16}$, Kay Tee Khaw ${ }^{17}$, Anne May ${ }^{18}$, Peter Nilsson ${ }^{19}$, Teresa Norat ${ }^{20}$, Kim Overvad ${ }^{21}$, Domenico Palli ${ }^{5}$, Salvatore Panico ${ }^{22}$, Jose Ramón Quirós ${ }^{23}$, Fulvio Ricceri ${ }^{24}$, Maria-Jose Sanchez ${ }^{25}$, Nadia Slimani ${ }^{26}$, Anne Tjonneland $^{27}$, Rosario Tumino ${ }^{28}$, Edith Feskens ${ }^{29}$, Elio Riboli ${ }^{20}$, Ulf Ekelund $^{1}$, Nick Wareham ${ }^{1}$

\section{Affiliations}

1. MRC Epidemiology Unit, Institute of Metabolic Science, Addenbrookes Hospital, Cambridge, UK

2. Department of Public Health and Clinical Medicine, Umea University, Umea, Sweden

3. Department of Epidemiology, Murcia Regional Health Authority, Murcia, Spain

4. Consortium for Biomedical Research in Epidemiology and Public Health (CIBER Epidemiología y Salud Pública-CIBERESP), Spain

5. Molecular and Nutritional Epidemiology Unit, ISPO, Cancer Prevention and Research Institute, Florence, Italy

6. Department of Epidemiology, Deutsches Institut für Ernährungsforschung Potsdam-Rehbrücke, Germany

7. National Institute for Public Health and the Environment, Bilthoven, The Netherlands

8. Institute of Community Medicine, University of Troms $\varnothing$, Troms $\varnothing$, Norway

9. Hellenic Foundation, Athens, Greece 
10. INSERM, CESP Center for Research in Epidemiology and Population Health, U1018, Nutrition, Hormones and Women's Health, Villejuif, France

11. Subdirección de Salud Pública de Gipuzkoa, Gobierno Vasco, San Sebastian, Spain

12. Navarra Public Health Institute, Pamplona, Spain.

13. Unit of Nutrition, Environment and Cancer, Cancer Epidemiology Research Program, Catalan Institute of Oncology, Barcelona, Spain

14. Nutritional Epidemiology Unit, Department of Preventive \& Predictive Medicine, Fondazione IRCCS Istituto Nazionale dei Tumori, Milan, Italy

15. Division of Cancer Epidemiology, German Cancer Research Centre, Heidelberg, Germany

16. Cancer Epidemiology Unit, University of Oxford, Oxford, UK

17. Clinical Gerontology Unit, University of Cambridge, Cambridge, UK

18. Julius Center for Health Sciences and Primary Care, Dept of Epidemiology, University Medical Center Utrecht, Utrecht, The Netherlands

19. Dept of Clinical Sciences, Lund University, Malmö, Sweden

20. Department of Epidemiology and Public Health, Imperial College London

21. Dept of Epidemiology, Institute of Public Health, Aarhus University, Aarhus, Denmark

22. Dipartimento di Medicina Clinica e Sperimentale, Universita di Napoli, Federico II, Naples, Italy

23. Asturias Council for Health \& Health Services, Spain

24. Hugef Foundation, Torino, Italy

25. Escuela Andaluza de Salud Pública, CIBER de Epidemiología y Salud Pública, Granada, Spain

26. Dietary Exposure Assessment Group, IARC, Lyon, France

27. The Danish Cancer Society, Institute of Cancer Epidemiology, Copenhagen, Denmark

28. U.O.S. Registro Tumori e U.O.C. Anatomia Patologica, Ospedale "Civile, M.P.Arezzo" ASP 7, Ragusa, Italy

29. Division of Human Nutrition - Section Nutrition and Epidemiology, University of Wageningen, Wageningen, The Netherlands

\section{References}

1. Bauman AE. Updating the evidence that physical activity is good for health: an epidemiological review 2000-2003. J Sci Med Sport. 2004;7(1 Suppl):6-19.

2. Craig CL, Marshall AL, Sjostrom M, Bauman AE, Booth ML, Ainsworth BE, et al. International physical activity questionnaire: 12-country reliability and validity. Med Sci Sports Exerc. 2003; 35:1381-95.

3. Wareham NJ, Jakes RW, Rennie KL, Schuit J, Mitchell J, Hennings $\mathrm{S}$, et al. Validity and repeatability of a simple index derived from the short physical activity questionnaire used in the European Prospective Investigation into Cancer and Nutrition (EPIC) study. Public Health Nutr. 2003;6:407-13.

4. Wareham NJ, Jakes RW, Rennie KL, Mitchell J, Hennings S, Day NE. Validity and repeatability of the EPIC-Norfolk physical activity questionnaire. Int J Epidemiol. 2002;31:168-74.

5. Jobe JB, Mingay DJ. Cognitive research improves questionnaires. Am J Public Health. 1989;79:1053-5.

6. Durante R, Ainsworth BE. The recall of physical activity: using a cognitive model of the question-answering process. Med Sci Sports Exerc. 1996;28:1282-91.

7. Riboli E, Hunt KJ, Slimani N, Ferrari P, Norat T, Fahey M, et al. European Prospective Investigation into Cancer and Nutrition (EPIC): study populations and data collection. Public Health Nutr. 2002;5(6(B)):1113-24.

8. Haftenberger M, Schuit AJ, Tormo MJ, Boeing H, Wareham N, Bueno-de-Mesquita HB, et al. Physical activity of subjects aged 50-64 years involved in the European Prospective Investigation into Cancer and Nutrition (EPIC). Public Health Nutr. 2002; 5(6(B)):1163-76.

9. Pols MA, Peeters PH, Ocke MC, Slimani N, Bueno-de-Mesquita $\mathrm{HB}$, Collette HJ. Estimation of reproducibility and relative validity of the questions included in the EPIC physical activity questionnaire. Int J Epidemiol. 1997;26(Suppl 1):S181-9.

10. Cust AE, Smith BJ, Chau J, van der Ploeg HP, Friedenreich CM, Armstrong BK, et al. Validity and repeatability of the EPIC physical activity questionnaire: a validation study using accelerometers as an objective measure. Int $\mathbf{J}$ Behav Nutr Phys Act. 2008;5:33.

11. Rose GA. The diagnosis of ischaemic heart pain and intermittent claudication in field surveys. Bull World Health Organ. 1962;27: 645-58.

12. Shephard RJ. PAR-Q, Canadian home fitness test and exercise screening alternatives. Sports Med. 1988;5:185-95.

13. Brage S, Brage N, Franks PW, Ekelund U, Wareham NJ. Reliability and validity of the combined heart rate and movement sensor actiheart. Eur J Clin Nutr. 2005;59:561-70.

14. Brage S, Ekelund U, Brage N, Hennings MA, Froberg K, Franks $\mathrm{PW}$, et al. Hierarchy of individual calibration levels for heart rate and accelerometry to measure physical activity. J Appl Physiol. 2007;103:682-92.

15. Stegle O, Fallert SV, MacKay DJ, Brage S. Gaussian process robust regression for noisy heart rate data. IEEE Trans Biomed Eng. 2008;55:2143-51.

16. Brage S, Brage N, Franks PW, Ekelund U, Wong MY, Andersen $\mathrm{LB}$, et al. Branched equation modeling of simultaneous accelerometry and heart rate monitoring improves estimate of directly measured physical activity energy expenditure. J Appl Physiol. 2004;96:343-51.

17. Henry CJ. Basal metabolic rate studies in humans: measurement and development of new equations. Public Health Nutr. 2005;8(7(A)):1133-52.

18. Ainsworth BE, Haskell WL, Whitt MC, Irwin ML, Swartz AM, Strath SJ, et al. Compendium of physical activities: an update of activity codes and MET intensities. Med Sci Sports Exerc. 2000;32(9 Suppl):S498-504.

19. Besson H, Ekelund U, Luan J, May AM, Sharp S, Travier N, et al. A cross-sectional analysis of physical activity and obesity indicators in European participants of the EPIC-PANACEA study. Int J Obes. 2009;33:497-506.

20. Ekelund U, Besson H, Luan J, May AM, Sharp SJ, Brage S, et al. Physical activity and gain in abdominal adiposity and body weight: prospective cohort study in 288,498 men and women. Am J Clin Nutr. 2011;93:826-35.

21. Khaw KT, Jakes R, Bingham S, Welch A, Luben R, Day N, et al. Work and leisure time physical activity assessed using a simple, 
pragmatic, validated questionnaire and incident cardiovascular disease and all-cause mortality in men and women: The European prospective investigation into Cancer in Norfolk prospective population study. Int J Epidemiol. 2006;35:1034-43.

22. Livingstone MB, Prentice AM, Coward WA, Ceesay SM, Strain JJ, McKenna PG, et al. Simultaneous measurement of free-living energy expenditure by the doubly labeled water method and heart-rate monitoring. Am J Clin Nutr. 1990;52:59-65.

23. Strath SJ, Brage S, Ekelund U. Integration of physiological and accelerometer data to improve physical activity assessment. Med Sci Sports Exerc. 2005;37(11 Suppl):S563-71.

24. Crouter SE, Churilla JR, Bassett DR Jr. Accuracy of the Actiheart for the assessment of energy expenditure in adults. Eur J Clin Nutr. 2008;62:704-11.
25. Thompson D, Batterham AM, Bock S, Robson C, Stokes K. Assessment of low-to-moderate intensity physical activity thermogenesis in young adults using synchronized heart rate and accelerometry with branched-equation modeling. J Nutr. 2006; 136:1037-42.

26. Assah FK, Ekelund U, Brage S, Mbanya JC, Wareham NJ. Accuracy and validity of a combined heart rate and motion sensor for the measurement of free-living physical activity energy expenditure in adults in Cameroon. Int $\mathbf{J}$ Epidemiol. 2011;40: 112-20.

27. Buchowski MS, Choi L, Majchrzak KM, Acra S, Mathews CE, Chen KY. Seasonal changes in amount and patterns of physical activity in women. J Phys Act Health. 2009;6:252-61. 